\title{
Long-Read Genome Sequence Resource of Ascochyta versabilis Causing Leaf Spot Disease in Pseudostellaria heterophylla
}

\author{
Yunbo Kuang, ${ }^{1,2}$ Zhe Wang, ${ }^{1}$ Felix Abah, ${ }^{3}$ Hongli Hu, ${ }^{3}$ Baohua Wang, ${ }^{3}$ Zonghua Wang, ${ }^{1,4}$ Huiping \\ Zhang, ${ }^{1}$ Zuyun $\mathrm{Ye}^{2, \dagger}$ and Jiandong Bao ${ }^{1 . \dagger}$ \\ ${ }^{1}$ Fujian University Key Laboratory for Plant-Microbe Interaction, College of Life Sciences, Fujian \\ Agriculture and Forestry University, Fuzhou 350002, China \\ ${ }^{2}$ The Engineering Technology Research Center of Characteristic Medicinal Plants of Fujian, College of \\ Life Sciences, Ningde Normal University, Ningde 352100, China \\ ${ }^{3}$ College of Plant Protection, Fujian Agriculture and Forestry University, Fuzhou 350002, China \\ ${ }^{4}$ Institute of Oceanography, Minjiang University, Fuzhou 350108, China
}

\begin{abstract}
Ascochyta versabilis is the fungal pathogen that causes the severe leaf spot disease of Pseudostellaria heterophylla (Miq.) Pax, a vital Chinese herbal plant. Here, we deployed PacBio single-molecule real-time long-read sequencing technology to generate a nearcomplete genome assembly for the $A$. versabilis $\mathrm{KC} 1$ strain and obtained a total of 9.80 $\mathrm{Gb}$ raw reads. These reads were processed into a $41.05 \mathrm{Mb}$ genome assembly containing 95 contigs with $\mathrm{N} 50$ of $1.70 \mathrm{Mb}$ and a maximum length of $3.93 \mathrm{Mb}$. A total of 10,457 gene models, of which 1,004 encode putatively secreted proteins, were identified in the genome. This highquality genome assembly and gene annotation resource will facilitate the institution of functional genetic studies aimed at providing a better insight into the infection mechanisms of $A$. versabilis to support the development of effective control strategies for leaf spot disease of $P$. heterophylla.
\end{abstract}

\section{Genome Announcement}

Ascochyta versabilis is the pathogen causing severe leaf spot disease of Pseudostellaria heterophylla (Miq.) Pax, an important traditional Chinese herb with several health benefits, including antitussive activity (Pang et al. 2011), ability to alleviate fatigue (Sheng et al. 2011), and as an antioxidant ( $\mathrm{Ng}$ et al. 2004). Annual yield losses caused by this pathogen are consistently high due to the absence of $A$. versabilis-resistant plant cultivars ( $L i$ et al. 2013; Wang et al. 1997).

The pathogenic fungus of leaf spot disease of $P$. heterophylla was first isolated in 1997 and was subsequently classified as a member of the genus Phoma (Wang et al. 1997). However, it was recently reclassified as $A$. versabilis based on morphological features and phylogenetic characterization of internal transcribed spacer regions 1 and 2, including the 5.8S nuclear ribosomal DNA gene (Li et al. 2018).

Although the leaf spot fungus represents a significant threat to the commercial cultivation of $P$. heterophylla, the molecular mechanisms regulating the pathogenesis of $A$. versabilis are

\footnotetext{
${ }^{\dagger}$ Corresponding authors: J. Bao; baojd@ fafu.edu.cn and Z. Ye; zyye0593@ndnu.edu.cn
}

The author(s) declare no conflict of interest.

Accepted for publication 13 September 2020.

(C) 2020 The American Phytopathological Society

\section{Funding}

This research is supported by National Key R\&D Program-Research Projects on Modernization of Traditional Chinese Medicine (2019YFC1710500), Natural Science Foundation of Fujian Province, China (2019J01385, 2020J0113340), Outstanding Youth Scientific Research Project of Fujian Agriculture and Forestry University (XJQ201511), Scientific Key Project of Ningde Normal University (2019ZDK18).

\section{Keywords}

Ascochyta versabilis, fungal pathogen, genome, leaf spot disease 
still unknown, owing to the absence of genome resources to support the identification and elucidation of pathogenesis mechanisms of $A$. versabilis.

To generate a high-quality genome assembly resource data for $A$. versabilis, the $A$. versabilis $\mathrm{KC} 1$ strain was isolated from a fungus-infected leaf spot on $P$. heterophylla from the Fujian province of China. High-quality genomic DNA of the strain KC1 was extracted from mycelia, following the CTAB method (Weiland 1997), and was sequenced using singlemolecule real-time (SMRT) sequencing technology developed by PacBio at the Novogene Bioinformatics Technology Co. Ltd. (Beijing). A single SMRT cell (1M V3) was used to sequence a $20-\mathrm{kb}$-long insert genomic library by the PacBio Sequel System. After filtering the low-quality raw reads, we obtained $9.80 \mathrm{~Gb}$ of raw reads with an average length of $10.23 \mathrm{~kb}$ and $\mathrm{N} 50$ of $14.37 \mathrm{~kb}$. The maximum read length was $64.70 \mathrm{~kb}$, and the average read accuracy was $86.24 \%$ (Table 1).

The genome of $A$. versabilis KC1 strain was de novo assembled with the HGAP4 pipeline of SMRT Link (v5.1.0, default parameters except expected genome length $=45 \mathrm{Mb}$ ). Initially, the longest reads ( $30 \%$ of the total, length cutoff $=25 \mathrm{~kb}$ ) were selected as seed reads, were corrected with the remaining long reads, and were then used to generate a draft assembly. To improve the continuity of assembly and fix base errors and gaps, $95.71 \%$ of the raw reads (229x coverage) were mapped to the draft assembly and generated a polished genome assembly with the Arrow algorithm. Removing organelle-originating contigs, we obtained a $41.05 \mathrm{Mb}$ assembly consisting of 95 contigs with $\mathrm{N} 50$ of $1.70 \mathrm{Mb}$ and maximum contig length of 3.93 Mb (Table 1). Genome completeness was assessed with BUSCO v4.05 (Seppey et al. 2019). The result showed that the assembly contains $752(99.21 \%)$ and 1,693 (99.24\%) complete orthologs in fungi $(n=758)$ and Ascomycota $(n=1,706)$, respectively (Table 1$)$.

Repeats were identified by RepeatModeler v1.08 (available online) and the genome sequence was masked by RepeatMasker v4.0.9 (available online). Notably, $13.93 \%$ of the genomic sequences are repetitive sequences. The soft-masking (with repeat regions into lower case letters) assembly was used to perform gene prediction using BRAKER2 (available online) (Hoff et al. 2019). Integrating ab initio and close homologous protein-based methods, a total of 10,457 protein-coding gene models were identified (Table 1). BUSCO assessment of gene predictions showed 98.28 and $96.42 \%$ completeness with 745 and 1,642 complete

Table 1. Genome characteristics ${ }^{a}$

\begin{tabular}{|c|c|}
\hline Features & Strain $\mathrm{KC}^{\mathrm{b}}$ \\
\hline Sequencing platform & PacBio Sequel \\
\hline Total reads (Gb) & 9.80 \\
\hline Average read length $(\mathrm{kb})$ & 10.23 \\
\hline Reads N50 (kb) & 14.37 \\
\hline Maximum read length $(\mathrm{kb})$ & 64.70 \\
\hline Average read accuracy & $86.24 \%$ \\
\hline Coverage & $229 x$ \\
\hline Assembly size (Mb) & 41.05 \\
\hline GC content & $49.62 \%$ \\
\hline Contig number & 95 \\
\hline Contig N50 (Mb) & 1.70 \\
\hline Contig L50 & 9 \\
\hline Average contig length $(\mathrm{Mb})$ & 0.43 \\
\hline Maximum contig length $(\mathrm{Mb})$ & 3.93 \\
\hline Protein-coding genes & 10,457 \\
\hline Candidate secreted proteins & 1,004 \\
\hline Repeat sequence & $13.93 \%$ \\
\hline $\begin{array}{l}\text { Genes annotated by Pfam, GO, KEGG, CAZy and } \\
\text { eggNOG }\end{array}$ & 8,368 \\
\hline Genome BUSCO assessment in fungi $(n=758)$ & C: 752 [S: 750, D: 2], F: 1, M: 5 \\
\hline $\begin{array}{l}\text { Genome BUSCO assessment in Ascomycota }(n= \\
1,706)\end{array}$ & C: 1,693 [S: $1,690, \mathrm{D}: 3], \mathrm{F}: 2, \mathrm{M}: 11$ \\
\hline Gene BUSCO assessment in fungi $(n=758)$ & C: 745 [S: 745, D: 0], F: 6, M: 7 \\
\hline Gene BUSCO assessment in Ascomycota $(n=1,706)$ & C: 1,642 [S: 1,637, D: 5], F: 26, M: 38 \\
\hline
\end{tabular}


orthologs in fungi $(n=758)$ and Ascomycota $(n=1,706)$, respectively (Table 1). Classically secreted proteins (with a signal peptide in the $\mathrm{N}$ terminus) were predicted per our previous pipeline (Bao et al. 2017), and 1,004 candidate secreted proteins were identified (Table 1). Also, a total of 8,368 (80\%) genes were annotated using Pfam, Gene Ontology, CAZy, Kyoto Encyclopedia of Genes and Genomes, and eggNOG databases (Table 1).

The nearly complete genome assembly and gene annotations data have been deposited at GenBank under the accession number JAAROD000000000 (BioProject: PRJNA613783).

\section{Acknowledgments}

We thank J. Norvienyeku for assistance with manuscript preparation.

\section{Author-Recommended Internet Resources}

BRAKER2: https://github.com/Gaius-Augustus/BRAKER

CAZy database: http://www.cazy.org

eggnog: http://eggnog5.embl.de

Gene Ontology resource: http://geneontology.org

Kyoto Encyclopedia of Genes and Genomes database: https://www.kegg.jp

RepeatModeler v1.08: http://www.repeatmasker.org/RepeatModeler

RepeatMasker v4.0.9: http://www.repeatmasker.org

Pfam: http://pfam.xfam.org

\section{Literature Cited}

Bao, J., Chen, M., Zhong, Z., Tang, W., Lin, L., Zhang, X., Jiang, H., Zhang, D., Miao, C., Tang, H., Zhang, J., Lu, G., Ming, R., Norvienyeku, J., Wang, B., and Wang, Z. 2017. PacBio sequencing reveals transposable elements as a key contributor to genomic plasticity and virulence variation in Magnaporthe oryzae. Mol. Plant 10:1465-1468.

Hoff, K. J., Lomsadze, A., Borodovsky, M., and Stanke, M. 2019. Whole-genome annotation with BRAKER. Methods Mol. Biol. 1962:65-95.

Li, D. Q., Xia, Z. M., Shao, C. Y., and Qin, Z. Y. 2013. Investigation and control of harmful organisms of Pseudostellaria heterophylla in Yuqing. J. Mountain Agric. Biol. 32:427-431.

Li, S. J., Zhou, X. L., and Yang, Y. L. 2018. Pathogen identification of leaf spot on Pseudostellaria heterophylla and screening of fungicides for its control. Plant Prot. 44:182-185.

Ng, T. B., Liu, F., and Wang, H. X. 2004. The antioxidant effects of aqueous and organic extracts of Panax quinquefolium, Panax notoginseng, Codonopsis

pilosula, Pseudostellaria heterophylla and Glehnia littoralis. J. Ethnopharmacol. 93:285-288

Pang, W., Lin, S., Dai, Q., Zhang, H., and Hu, J. 2011. Antitussive activity of Pseudostellaria heterophylla (Miq.) Pax extracts and improvement in lung function via adjustment of multi-cytokine levels. Molecules 16:3360-3370.

Seppey, M., Manni, M., and Zdobnov, E. M. 2019. BUSCO: Assessing genome assembly and annotation completeness. Methods Mol. Biol. 1962:227-245.

Sheng, R., Xu, X., Tang, Q., Bian, D., Li, Y., Qian, C., He, X., Gao, X., Pan, R., Wang, C., Luo, Y., Xia, Y., and Dai, Y. 2011. Polysaccharide of Radix Pseudostellariae improves chronic fatigue syndrome induced by poly I:C in mice. Evid.-Based Compl. Alt. 2011:840516.

Wang, Z. H., Tang, J. Z., Wu, S. J., Wu, J. H., Zhang, J. N., and Wang, B. H. 1997. A new disease of Pseudostellaria heterophylla in Zherong. Acta Phytopathologica Sin. 37:174

Weiland, J. J. 1997. Rapid procedure for the extraction of DNA from fungal spores and mycelia. Fungal Genet. Rep. 44:22. 